\title{
An Introduction to OpenStreetMap in Geographic Information Science: Experiences, Research, and Applications
}

\author{
Jamal Jokar Arsanjani, Alexander Zipf, Peter Mooney \\ and Marco Helbich
}

\begin{abstract}
Recent years have seen new ways of collecting geographic information via the crowd rather than organizations. OpenStreetMap (OSM) is a prime example of this approach and has brought free access to a wealth of geographic information - for many parts of the world, for the first time. The strong growth in the last few years made more and more people consider it as a potential alternative to commercial or authoritative data. The increasing availability of ever-richer data sets of freely available geographic information led to strong interest of researchers and practitioners in the usability of this data - both its limitations and potential. Both the unconventional way the data is being produced as well as its richness and heterogeneity have led to a range of different research questions on how we can assess, mine, enrich, or just use this data in different domains and for a wide range of applications. While this book cannot present all types of research around OpenStreetMap or even the broader category of User Generated Content (UGC) or Volunteered Geographic Information (VGI), it attempts to provide an overview of the current state of the art by presenting some typical and recent examples of work in GIScience on OSM. This chapter provides an introduction to the scholarly work on OpenStreetMap and its current state and summarizes the contributions to this book.
\end{abstract}

J. Jokar Arsanjani ( $₫)$. A. Zipf

GIScience Research Group, Institute of Geography, Heidelberg University,

69120 Heidelberg, Germany

e-mail: jokar.arsanjani@geog.uni-heidelberg.de
A. Zipf
e-mail: zipf@uni-heidelberg.de
P. Mooney
Department of Computer Science, Maynooth University,
Maynooth, Co. Kildare, Ireland
e-mail: peter.mooney@nuim.ie

M. Helbich

Department of Human Geography and Spatial Planning, Utrecht University,

Heidelberglaan 2, 3584 CS Utrecht, The Netherlands

J. Jokar Arsanjani et al. (eds.), OpenStreetMap in GIScience,

Lecture Notes in Geoinformation and Cartography,

DOI 10.1007/978-3-319-14280-7_1 


\section{Introduction}

Access to spatial data and cartographic products has changed radically over the last decade or so. Traditionally, governmental agencies, cartographic centers, and commercial agencies were the only sources for end-users seeking spatial data. One of the most formidable barriers to more widespread access to these geodata were created by often prohibitive high fees and license charges in combination with time- and purpose-limited copyright restrictions imposed. This business model was rather successful, but made access to high-quality geodata very difficult for all but a small number of end users. Changes in Information and Communication Technology (ICT) brought about by the Internet and social media and the availability of inexpensive portable satellite navigation devices has seen this traditional geodata business model challenged. One of the key driving forces in this change has been the OpenStreetMap (OSM) project. OSM was launched in 2004 with the mission of creating an editable map of the whole world and released with an open content license (http://wiki.openstreetmap.org/wiki/About). In general, OSM aims at building and maintaining a free editable map database of the world in a collaborative manner so that people and end-users are not forced to buy geodata in the traditional way and subsequently be subjected to restrictive copyright and license commitments. OSM started initially with a focus on mapping streets and roads. Since then it has moved far beyond these entities and it now contains a very rich variety of geographical objects (e.g., buildings, land use, Points of Interest) from all over the planet being mapped by thousands of volunteer contributors to the project. Aside from the obvious commercial benefits offered by OSM, the project has revolutionized the way in which geodata is collected. No longer are the collection of geodata and the development of cartographic products limited to specialists, geographic surveyors, or cartographers.

OSM is often referred to as the Wikipedia map of the world. As it is built on many of the same ICT structures as Wikipedia it offers its project contributors the possibility of (a) almost immediate updating of the map database as well as very frequent updating of associated editing software and other tools; (b) importing geodata recorded from Global Positioning System (GPS)-enabled devices, smartphones, and other digital maps tools; (c) access to the full history of mapping activities in OSM over its lifetime; and finally (d) collaboration with other OSM users and contributors through various communication channels including mailing lists, discussion forums, and physical meetings (Mooney and Corcoran 2013a). The gradual evolution of the OSM ecosystem has been very successful. The project got off to a slow start but since 2007 there has been an ever-increasing rate of people joining the project. In November 2014, OSM had approximately 1.85 million registered users and contributors (http://wiki.openstreetmap.org/wiki/Stats). As mentioned previously, the era of ubiquitous Internet, social media, open-source software, etc. has seen many citizen knowledge-based projects for a host of diverse purposes launched on the Internet over the last few years. OSM has been a unique case. The academic and industrial communities have recognized OSM not solely 
based on its rise to become an important distributor of geodata but its wider success in growing a global community of people willing to participate in the collection and maintenance of geodata. The OSM community is actively involved in much more than collecting geodata to build and maintain this global geodatabase. In addition, the community is involved in, for example, humanitarian work, open source software development to support OSM and the GIS community, and in building a network of support for those using and contributing to the OSM project.

In recent years, several scientific disciplines (e.g. geography, GIScience, spatial planning, cartography, computer science, and ecology) have realized the immense potential of OSM and it has become the subject of academic research. OSM offers researchers a unique dataset that is global in scale and a body of knowledge created and maintained by a very large collaborative network of volunteers. Research on OSM has shown that its geodata in some parts of the world are more complete and locationally and semantically more accurate than the corresponding proprietary datasets (e.g., Zielstra and Zipf 2010; Neis and Zipf 2012; Helbich et al. 2012), while being of high spatial heterogeneity. Skepticism amongst the GIS community and industry surrounding the quality of the geodata in OSM has seen a major effort being made on evaluating the quality of the OSM geodata. This has a led to the development of a number of software tools and methodologies for analyzing the quality (Roick et al. 2011; Helbich et al. 2012; Jokar Arsanjani et al. 2013a; Jokar Arsanjani and Vaz 2015c). Other approaches even try to improve the OSM data through algorithms dedicated to specific object types, such as addresses for geocoding (Amelunxen 2010). Investigation of the development and evolution of OSM across the globe over time has also emerged as a research topic for many academic studies (Mooney et al. 2012; Neis and Zipf 2012; Jokar Arsanjani et al. 2013c; Mooney and Corcoran 2013; Fan et al. 2014).

Extracting value-added information from the OSM database has become another emerging research topic for researchers to attempt to understand OSM better (Hagenauer and Helbich 2012; Mooney and Corcoran 2012; Mooney et al. 2013; Jokar Arsanjani et al. 2015). Hagenauer and Helbich (2012), for instance, predicted missing urban areas through artificial neural networks. Bakillah et al. (2014a) derived population estimations from OSM and an emerging important topic is land use maps that can be generated using OSM (Jokar Arsanjani et al. 2014; Jokar Arsanjani and Vaz 2015c). Klonner et al. (2014) investigated the updating of Digital Elevation Models and Fan et al. (2014) estimated building types from OSM.

Both inside and outside of the academic sphere, OSM is now being used increasingly in a variety of practical or scientific applications in different domains, which demonstrates the usability of the crowdsourced geodata in OSM. However, in all of these cases the characteristics of OSM must be considered. Because of the flexibility and open data-like structure of OSM, it is possible to use or even adapt and improve OSM for a large range of purpose-directed applications, as we will see below. As mentioned above, there are some data quality issues with the OSM database which can be mitigated against through specialized approaches to using the actual geodata (Goodchild 2013). This has brought about a host of examples of applications and domain-specific research. A first important category is the 
development of a set of different special routing and navigation systems that operate on a large scale. Examples include: routing for cars, bikes, and pedestrians such as in OpenRouteService, (Schmitz et al. 2008); emergency routing (Neis et al. 2010); wheelchair routing (Neis 2014); emergency response and evacuation simulation (Bakillah et al. 2012); indoor routing (Goetz 2012); or agricultural logistics (Lauer et al. 2014). Further typical uses of OSM include improving cartography (Rylov and Reimer 2014) or developing Location Based Services (LBS) (Schilling et al. 2009). Another innovation was the development of 3D city models from OSM (Over et al. 2010; Goetz 2013). Further research has focused on attempting to extend the current OSM spatial data model by working on extensions such as: 3D (Goetz and Zipf 2012), indoor mapping (Goetz and Zipf 2012), or wheelchair routing (Neis 2014) and using the results from this in a range of applications.

The relationship between OSM and open data standards, in particular Spatial Data Infrastructures (SDI) and the future direction of the Web 2.0 paradigm, is a question still requiring further discussion. In particular, the large volumes of data being updated by the minute that are now available pose challenges with regards to their handling and keeping them up to date on a global scale.

The discussion in the preceding paragraphs has shown that OSM has now emerged as a new research area. It has the potential to bring disparate research disciplines together and enhances interdisciplinary and multidisciplinary investigations. This interdisciplinary research collaboration can contribute to a more profound and cross-disciplinary understanding of citizens' knowledge-based efforts in projects such as OSM. It also provides an interesting platform for the academic research community to collaborate with these communities towards interactive collection of up-to-date geodata from citizens by means of novel computationally oriented methods such as network analysis, machine learning, and computer simulation models. As the examples above have demonstrated, these practical investigations on OSM provide a rich set of opportunities to discover novel and valuable patterns inherent in the geodata collected by citizens, to better understand the activities of contributors to open knowledge projects, the characteristics of their human-computer interactions, and the potential to tackle classical GIS research questions using this modern and revolutionary approach to the collection and distribution of geographic data.

\section{A Short Overview of the OpenStreetMap Research Landscape}

In this section, we present a brief overview of the OSM research landscape through a word cloud approach. To do so, a search query was applied on 16 August 2014 in Google Scholar looking for four terms "OpenStreetMap", "OSM", "VGI", and "Volunteered Geographic Information" either in the abstracts, titles or keywords. In total, 224 documents were collected. The collection of titles, abstracts, and keywords were explored by means of word clouds. Word clouds provide an intuitive 


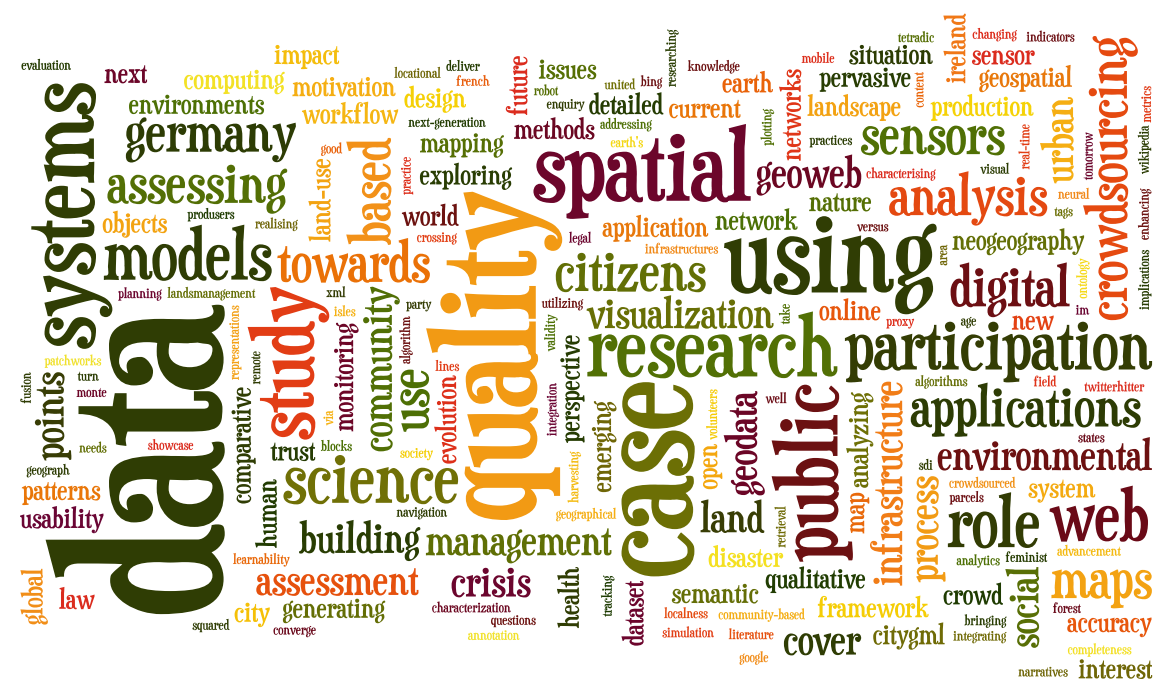

Fig. 1 Word cloud of the papers' titles

impression about common words and show the number of times a certain word appeared in the literature. This is expressed by varying font sizes. Larger font sizes refer to words that appear more often than smaller font sizes (Helbich et al. 2013). It should be noted that the aforementioned search terms were removed from the resulting word cloud as their usage frequencies were substantial and masked the other terms. We leave these figures for the readers to interpret them visually and gain some insights about the research on OSM so far.

As editors of this volume, we have been involved in research connected to OpenStreetMap for many years. From our own empirical experience, these word

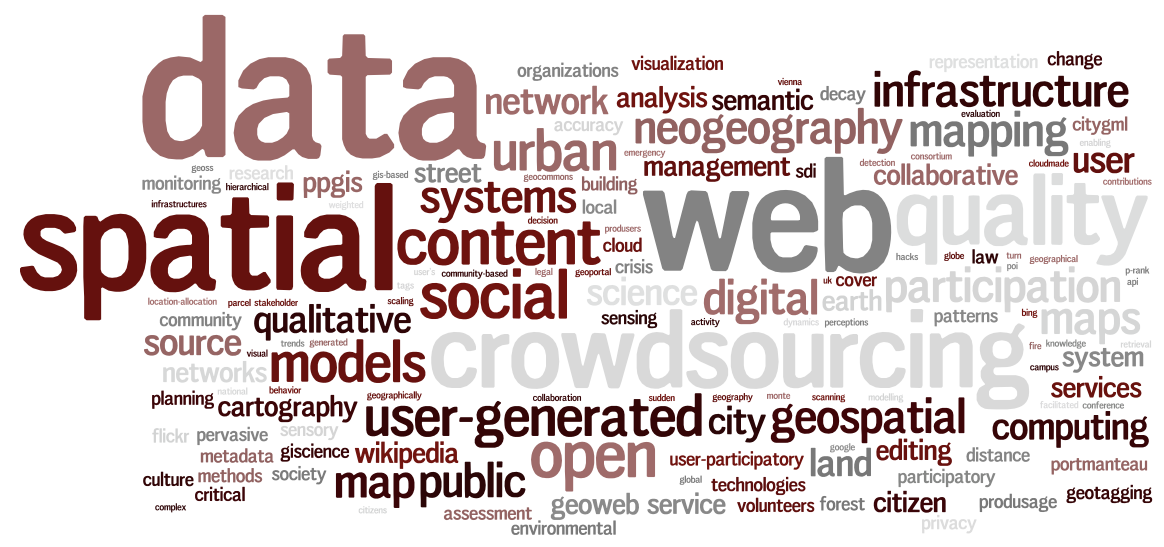

Fig. 2 Word cloud of the papers' keywords 


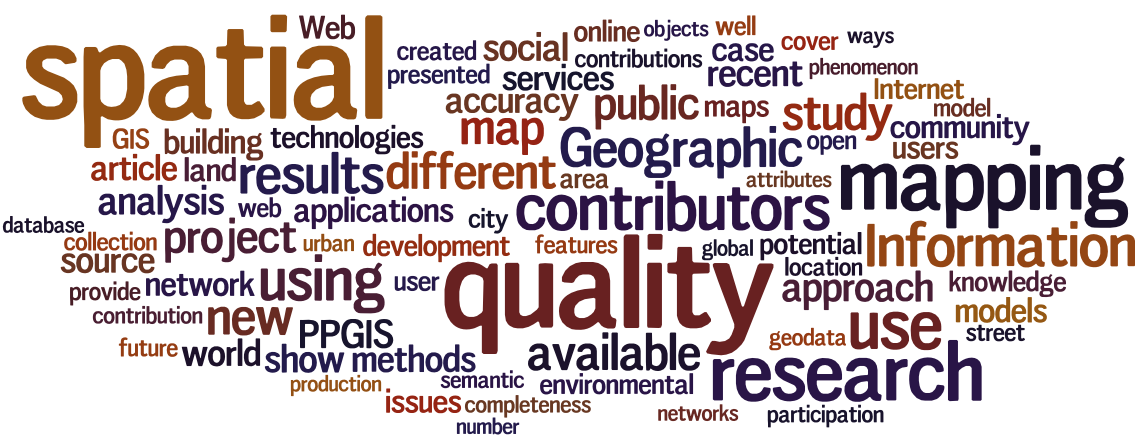

Fig. 3 Word cloud of the papers' abstracts

clouds capture the essence of academic research on OpenStreetMap from the past number of years. In Figs. 1 and 2 where the word clouds of paper title terms and keywords are presented we see a few dominant terms, particularly data, quality, research, systems, and participation. As we mentioned earlier, the issue of the quality of OSM data has been on the research agenda for many years now. It is likely to remain on the agenda for some time to come. However, these titles also reflect the expansion of OSM research to consider the citizen and volunteer participation, which drives the expansion of the project. The systems, models, sensors, and applications which collect, analyze, store, manage, transform, and distribute the data must now be studied and explored in more detail as OpenStreetMap grows in size potentially towards being considered as Geographic Big Data (Goodchild 2013).

The word cloud in Fig. 3 displays the most frequently occurring terms in the abstracts of the papers returned in our Google Scholar searches. Quality is a dominant term but we see the concepts of research, social, community, methods, and development emerging. There are obvious visual linkages to the word cloud in Fig. 4 where the abstracts of the chapters included in this volume are visualized.

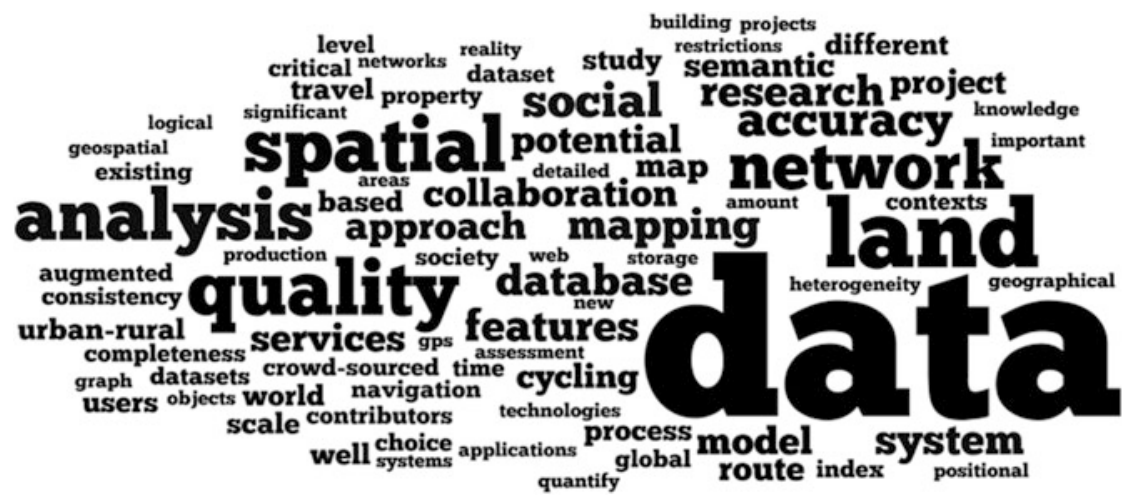

Fig. 4 Word cloud of our chapters' abstracts 
We can immediately see the same set of dominant terms. However, in our abstracts word cloud there is somewhat more diversity with urban modelling, navigation, modelling, and knowledge management related terms being highlighted.

\section{Geography of OpenStreetMap}

As already stated in the literature (e.g., Mooney and Corcoran 2012; Neis 2014), OpenStreetMap has its own geography across time and space. In other words, we rarely see identical patterns of contributions in two different regions/countries. When speaking of OpenStreetMap quality and contributions networks the importance of studying diverse case studies has been highlighted. Hence, in this section, two different maps are generated from the OSM statistics, which demonstrate the heterogeneity of OSM in different countries. Figure 5 displays the total number of created nodes in October 2014. This map displays a thematic categorization of created nodes, which is one of the key elements in measuring OSM contributions. It should be noted that in this comparative report, the size of the country, population, gross domestic product (GDP), and a number of other physical characteristics of the countries are not taken into consideration. However, they are of great importance in performing further in-depth analysis. For instance, the dominant land cover types in Canada and Australia should be excluded in considering the size of the country as apart from land cover there are no objects to be mapped and the contributed nodes have very likely occurred within urban areas. Besides, their populations are not comparable to the USA, China, and India.

Nonetheless, focusing on count gives an overall indication that the high number of node creation is not limited to European countries, but other countries are also

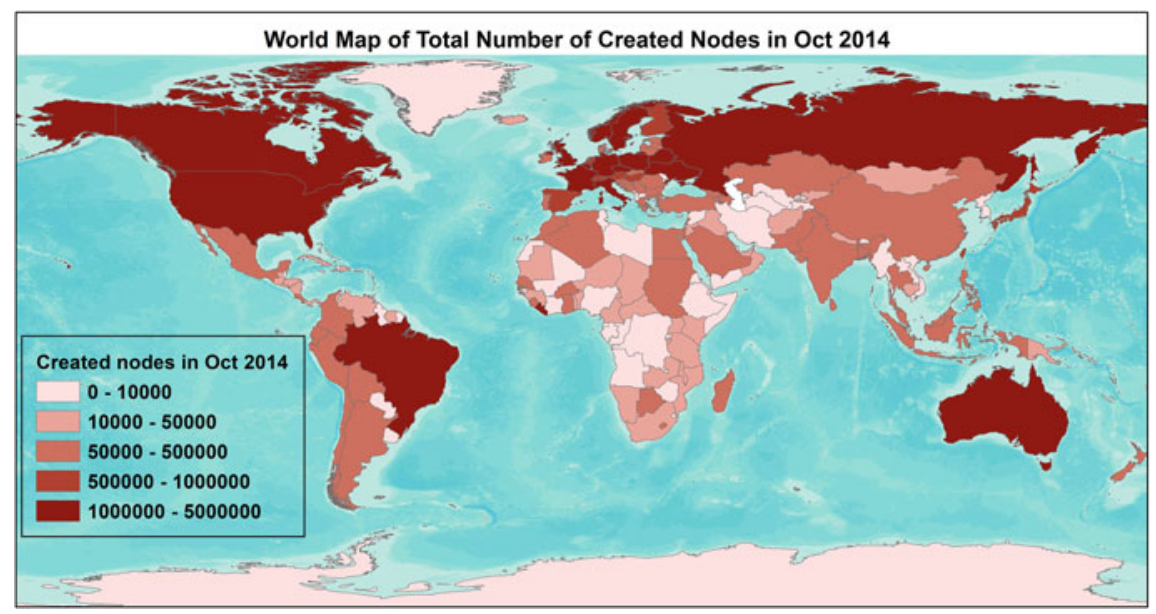

Fig. 5 A world map of the total created nodes in October 2014 
emerging in OSM. Amongst these emerging countries, north America including USA and Canada, south American countries particularly Brazil, Australia, and some Asian and African countries can be named, which calls for further studies in these regions to find out how actively and accurately mapping in OSM is being undertaken. In terms of number of created nodes, in total over 46 million nodes were created in this month. In a number of countries, no nodes were created. However, Germany, United States, Russia, Czech Republic, Italy, Poland, France, Norway, Liberia, Canada, and Japan received the most created nodes, respectively.

In terms of total active members, i.e., mappers, in this period, while in total 3,048 members logged into OSM, the majority of them were from Germany (535), United States (215), Russia (212), France (195), Italy (156), Poland (155), UK (128), Spain (96), Austria (81), and Japan (55). In order to normalize the number of active members, the average number of active members per day in October 2014 is divided by the total population of the countries in terms of millions of people in 2010. Figure 6 shows the average number of active members per day for October 2014 per million people. This map helps to detect the countries that have a large portion of their population involved in the mapping process in OSM. Italy, the Netherlands, Kuwait, Croatia, and Liberia were at the top of the list. It is interesting to see that a number of countries from all continents have more than 1 member per million population active in mapping. On the contrary, a number of Asian and African countries have a very minor proportion of their population involved in mapping. This confirms the empirical findings that only a small portion of the population is mapping. It is worth mentioning that this finding is based on our analysis within the chosen timeframe for sharing general impressions about OSM and activities in OSM certainly also has a temporal pattern, which is an important indicator to be considered.

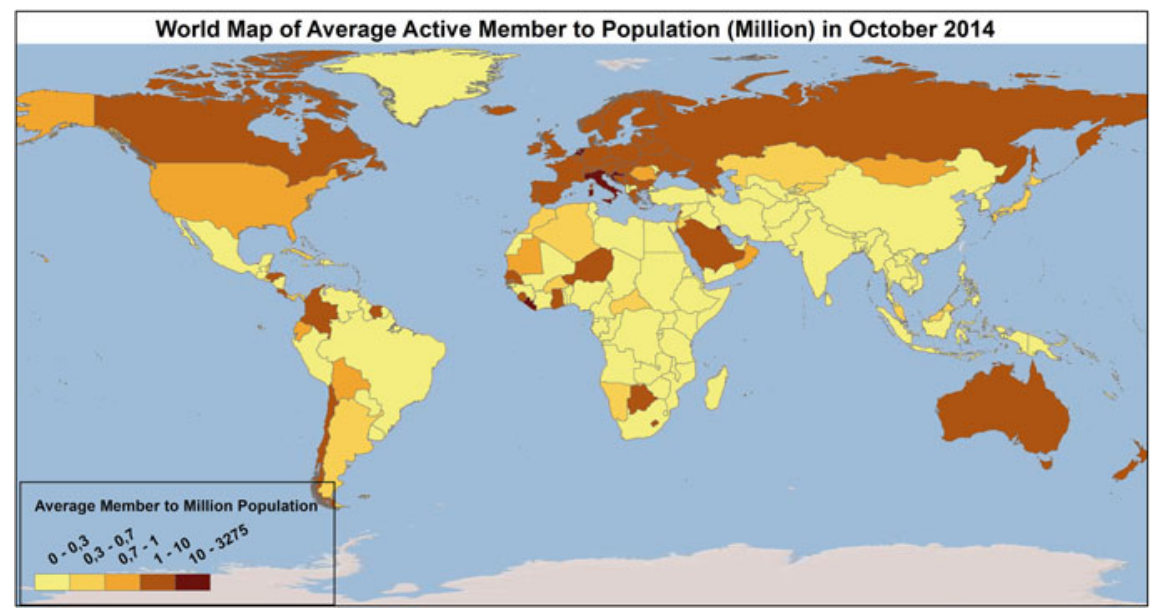

Fig. 6 A world map of average number of active members to population (million) in October 2014 
It is interesting to note that contrary to Germany as an active country in OSM, some other countries are emerging in OSM and there are still large gaps in the OSM data from these countries. However, this can be viewed positively. Information dissemination about OSM within the last few years has promoted additional people to become members of OSM. Slowly but surely OSM is gaining popularity in these countries. Perhaps OpenStreetMap is helping address the participation inequality that is strongly represented in many types of User-Generated Content on the Internet today. This "Digital Divide" indicates that very small groups with specific demographic and geographical characteristics are responsible for production of most of the UGC we see on the Internet today. However, these map visualizations indicate that OpenStreetMap is reaching into countries and regions which heretofore would have felt the consequences of the digital divide (Graham et al. 2012). Improvements in ICT infrastructures and IT education for socially deprived groups such as women and children coupled with more ubiquitous access to smartphone technology has provided an environment where participation in OpenStreetMap can increase. Research will need to be undertaken to gain a better understanding of the social processes involved in these changes.

\section{Objectives and Scope}

The present volume entitled "OpenStreetMap in Geographic Information Science: experiences, research, and applications" presents a collection of experiences and research which has been carried out with OSM as the central and core theme. The book seeks to build a firm foundation for research work focused on integrating OSM. Chapters will address the following research topics:

(a) State-of-the-art and cutting-edge approaches for data quality analysis in OSM and VGI.

(b) Investigations on understanding OSM contributors and the nature of their contributions.

(c) Identification of patterns of contributions and contributors.

(d) Applications of OSM in different domains.

(e) Mining value-added knowledge and information from OSM.

(f) Limitations in the analysis of OSM data.

(g) Integration of OSM with commercial and non-commercial geodatasets.

We expect that this book will deliver significant scientific outcomes, which will further stimulate international research networking and collaboration. As outlined above, the inherent cross-disciplinary essence of OSM research combined with the emerging data quality, data mining, and patterns determination approaches to analysis of OSM means that contributing scholars for this book will be expected to have a diverse academic background not limited to geographic information science, cartography, computer science, statistics, and sociology. We feel that these transand inter-disciplinary contributions permit a deeper understanding of how the OSM 
project works and has become the phenomenal success that it is today. Last, but not least, the book will strive to bring OSM into the core of GIScience where the diverse worlds of new and classical geography and cartography will meet.

This book presents some cutting-edge developments and applications in the field of geography, spatial statistics, geographic information science, social science, and cartography. This collection of chapters is highly relevant for, but not limited to, the following potential audience and readership: researchers, postgraduates, and professionals.

The high response to our call for chapters shows that the intention of this book to be widely announced has been fulfilled. By the end of January 2014, a total of 34 chapter proposals were submitted and after an internal review by the editors, 30 authors of those originally 34 submitted chapter proposals were invited to submit a full chapter manuscript. After the final chapter submission deadline on 30 May 2014, a total of 29 manuscripts were submitted. Thereafter, each of the 29 chapter manuscripts was evaluated through a double-blind review process by at least two or three international experts in the respective field. For the review process, the standard Springer review guidelines were applied. Besides the innovative aspect of the research, the scientific quality of the research weighted heavily on the decision as to whether or not a manuscript was accepted or rejected. After two rounds of reviews conducted by international experts, the editors made the decision on whether or not the manuscript was fit for publication. In October 2014, 14 chapters were accepted and along with one introductory chapter and one conclusive chapter are now included in the present book.

\section{Structure of the Book}

The book covers several areas of OSM, each associated with a main theme of the book. The present volume has the following four sections: (1) Data management and quality, (2) Social context, (3) Network modeling and routing, and (4) Land management and Urban form. However, this structure should not be understood as fixed and definitive. Quite the contrary, the boundaries between these sections are partly fuzzy and overlap each other to some extent.

Section 1 on Data Management and Quality includes five chapters. In chapter "Assessment of Logical Consistency in OpenStreetMap Based on the Spatial Similarity Concept", Peyman Hashemi and Rahim Ali Abbaspour propose an approach for assessing the logical consistency in OSM based on the concept of spatial similarity in multi-representation considering three elements, i.e. directional relationships, topological relationships, and metric distance relationships. Jokar Arsanjani et al. in chapter "Quality Assessment of the Contributed Land Use Information from OpenStreetMap Versus Authoritative Datasets" attempt to comparatively assess the accuracy of the contributed OSM land use features in four German metropolitan areas versus the pan-European GMESUA dataset as a reference. Their empirical findings suggest OSM to be alternative complementary 
source for extracting LU information with over half of the selected cities mapped by mappers. Moreover, the results identify which land types preserve high/moderate/ low accuracy across cities for urban land use mapping. The findings strengthen the potential of collaboratively collected LU features for providing temporal LU maps as well as updating or enriching existing inventories. Chapter "Improving Volunteered Geographic Information Quality Using a Tag Recommender System: The Case of OpenStreetMap" by Arnaud Vandecasteele and Rodolphe Devillers proposes an approach for both improving the semantic quality and reducing the semantic heterogeneity of VGI datasets through implementing a tag recommender system, called OSMantic plugin, that automatically suggests relevant tags to contributors during the editing process. Their approach helps contributors find the most appropriate tags for a given object, hence reducing the overall dataset semantic heterogeneity. This plugin is developed for the Java OSM editor (JOSM) and different examples illustrate how this plugin can be used to improve the quality of VGI data. In chapter "Inferring the Scale of OpenStreetMap Features", Guillaume Touya and Andreas Reimer propose and compare two concurrent approaches at automatically assigning scale to OSM objects. Their first approach is based on a multi-criteria decision making model, with a rationalist approach for defining and parameterizing the respective criteria, yielding five broad Level of Detail classes. Their second approach attempts to identify a single metric from an analysis process, which is then used to interpolate a scale equivalence. Both approaches are combined and tested against well-known CORINE data, resulting in an improvement of the scale inference process. The chapter closes with a presentation of the most pressing open problems. In chapter "Data Retrieval for Small Spatial Regions in OpenStreetMap", Roland M. Olbricht investigates what design choices are required to be able to answer almost any geographic query whilst serving common use cases fast enough such that the services based on this database are fast on affordable and standard sized hardware. He evaluates the usage patterns from the main instance of Overpass API on overpass-api.de by considering more than 40 million requests from 2012 and 2013.

Section 2 deals with Social Context and comprises of three chapters. In chapter "The Impact of Society on Volunteered Geographic Information: The Case of OpenStreetMap", Afra Mashhadi et al. address whether the society and its characteristics such as the socio-economic factors have an impact on what part of the physical world is being digitally mapped so that we can understand where crowdsourced map information can be relied upon. They measure the positional and thematic accuracy as well as the completeness of OSM data and quantify the role of society on the state of this digital production and finally quantify the effect of social engagement as a method of intervention for improving user participation. Georg Glasze and Chris Perkins frame a research agenda in chapter "Social and Political Dimensions of the OpenStreetMap Project: Towards a Critical Geographical Research Agenda" that draws upon critical cartography, but widens the scope of analysis to the assemblages of practices, actors, technologies, and norms at work: an agenda which is inspired by the critical GIS literature, to take the specific social contexts and effects of technologies into account, but which deploys a processual 
view of mapping. They recognize that a fundamental transition in mapping is taking place, and that OSM may well be of central importance in this process. In chapter "Spatial Collaboration Networks of OpenStreetMap", Klaus Stein et al. describe a new type of spatial collaboration network that can be extracted from OSM edit history data and show how to apply the measurement of interlocking responses known from research on non-spatial collaboration in wikis to collaboration in OSM. Finally, they discuss the advantages of their approach by demonstrating an analysis of collaboration on OSM sample data.

Section 3 deals with Network modeling and routing and includes three chapters. In chapter "Route Choice Analysis of Urban Cycling Behaviors Using OpenStreetMap: Evidence from a British Urban Environment", Godwin Yeboah and Seraphim Alvanides undertake a route choice analysis using the cyclingfriendly version of OSM as the transportation network for analysis, alongside GPS tracks and travel diary data for 79 Utility Cyclists around Newcastle upon Tyne in North East England. They suggest that OSM can provide a robust transportation network for cycling research, in particular when combined with GPS track data, and conclude that network restrictions for both observed and shortest paths are significant, suggesting that route directness is an important factor to be considered for restricted and unrestricted networks. Chapter "The Next Generation of Navigational Services Using OpenStreetMap Data: The Integration of Augmented Reality and Graph Databases" by Pouria Amirian et al. describes the implementation of a navigational application as part of the eCampus project in Maynooth University. The application provides users with several navigation services with navigational instructions through standard textual and cartographic interfaces and also through augmented images showing way-finding objects. Jorge Gil presents the process of building a multi-modal urban network model using OSM data in chapter "Building a Multimodal Urban Network Model Using OpenStreetMap Data for the Analysis of Sustainable Accessibility". He develops various algorithmic procedures to produce the network model, supporting the reproducibility of the process and addressing the challenges of using OSM data for this purpose and addresses the great potential of OSM for urban analysis, thanks to the detail of its attributes and its open and universal coverage.

Land management and urban form is the focus of the final Sect. 4, comprising of three chapters. Chapter "Assessing OpenStreetMap as an Open Property Map" by Mohsen Kalantari and Veha La provides an assessment of OSM as a crowdsourcing system in collecting and recording land tenure information using a case study in Victoria, Australia. Their chapter studies the completeness of the public property records in OSM, and the location, shape, area and description of the existing records, and finally discusses the potential of OSM as an Open Property Map. Jacinto Estima and Marco Painho in chapter "Investigating the Potential of OpenStreetMap for Land Use/Land Cover Production: A Case Study for Continental Portugal" review the existing literature on using OSM data for land use/cover database production and move this research forwards by exploring the suitability of the OSM Points of Interest. They conclude that OSM can greatly contribute to mapping specific land types. In chapter "Using Crowd-Sourced Data 
to Quantify the Complex Urban Fabric-OpenStreetMap and the Urban-Rural Index", Johannes Schlesinger presents an Urban-Rural Index (URI), which tackles the lack of any classification of the urban-rural continuum especially in regions of the world where accurate and up-to-date geodata is hardly available. His paper draws on the analysis of three study sites: Bamenda in Cameroon, Moshi in Tanzania, and Bangalore in India, and concludes that URI as a reproducible representation of the spatial complexity of the urban landscape and its surrounding areas has the potential to contribute to the understanding of urban development patterns.

In the final chapter (chapter "An Outlook for OpenStreetMap") by Peter Mooney, the future research perspective of research on OpenStreetMap is reviewed. In the chapter, he structures his future vision of OpenStreetMap research by using the content of this volume and other OSM literature as a basis for future work.

Last but not least, the editors express their appreciation to all reviewers for their kind support and their critical and constructive comments for each chapter. Undoubtedly, their efforts have significantly enriched the quality of the entire volume. We profoundly appreciate the efforts of all authors who submitted a full chapter manuscript and chose this book as their desired publication outlet for their research. Furthermore, we would like to thank Prof. Muki Haklay for his invited comment. Finally, Jamal Jokar Arsanjani thanks the Alexander von Humboldt foundation and the Institute of Geography at Heidelberg University, Germany, for providing the foundation for this book. Peter Mooney would like to thank the Environmental Protection Agency Ireland for funding support. Finally, we acknowledge the Springer team as well as the series editors William Cartwright, Georg Gartner, Liqiu Meng, and Michael Peterson for their great assistance throughout the whole publication process. Certainly, without all these helping hands, this volume would have never been published. The book is partially sponsored by the COST Action IC1203.

\section{References}

Amelunxen C (2010) On the suitability of volunteered geographic information for the purpose of geocoding. In: Car A, Griesebner G, Strobl J (eds) Proceedings of the geoinformatics forum salzburg, na. Retrieved http://koenigstuhl.geog.uniheidelberg.de/publications/2010/Amelunxen/ GI_Forum_Amelunxen.pdf

Bakillah M, Andrés Domínguez J, Zipf A, Liang SHL, Mostafavi MA (2013) Multi-agent evacuation simulation data model with social considerations for disaster management context. In: Zlatanova S, Peters R, Dilo A, Scholten H (eds) Intelligent systems for crisis management, SE-1. Springer, Berlin, Heidelberg, pp 3-16. doi:10.1007/978-3-642-33218-0_1

Bakillah M, Liang S, Mobasheri A, Jokar Arsanjani J, Zipf A (2014a) Fine-resolution population mapping using OpenStreetMap points-of-interest. Int J Geogr Inf Sci 28:1940-1963

Bakillah M, Lauer J, Liang S, Zipf A, Jokar Arsanjani J, Loos L, Mobasheri A (2014b) Exploiting big VGI to improve routing and navigation services. In: Big data techniques and technologies in geoinformatics, pp 177-192

Fan H, Zipf A, Fu Q, Neis P (2014) Quality assessment for building footprints data on OpenStreetMap. Int J Geogr Inf Sci 28:700-719. doi: 10.1080/13658816.2013.867495 
Goetz M (2013) Towards generating highly detailed 3D CityGML models from OpenStreetMap. Int J Geogr Inf Sci 27:845-865

Goetz M, Zipf A (2012) Using crowdsourced indoor geodata for agent-based indoor evacuation simulations. ISPRS Int J Geo-Inf 1(2):186-208. doi:10.3390/ijgi1020186

Goodchild MF (2013) The quality of big (geo) data. Dialogues Hum Geogr 3:280-284

Graham M, Hale S, Stephens M (2012) Digital divide: the geography of internet access. Environ Plan A 44:1009-1010

Hagenauer J, Helbich M (2012) Mining Urban land-use patterns from volunteered geographic information by means of genetic algorithms and artificial neural networks. Int J Geogr Inf Sci 26:963-982

Helbich M, Amelunxen C, Neis P (2012) Comparative spatial analysis of positional accuracy of OpenStreetMap and proprietary geodata. In: Jekel T, Car A, Strobl J, Griesebner G (eds) Geospatial crossroads @ GI_Forum 2012, Wichmann, Heidelberg, Salzburg, pp 24-33

Helbich M, Hagenauer J, Leitner M, Edwards R (2013) Exploration of unstructured narrative crime reports: an unsupervised neural network and point pattern analysis approach. Cartogr Geogr Inf Sci 40:326-336

Jokar Arsanjani J, Barron C, Bakillah M, Helbich M (2013a) Assessing the quality of OpenStreetMap contributors together with their contributions. In: 16th AGILE international conference on geographic information science, Leuven, Belgium, pp 14-17

Jokar Arsanjani J, Helbich M, Bakillah M, Hagenauer J, Zipf A (2013b) Toward mapping land-use patterns from volunteered geographic information. Int J Geogr Inf Sci 27: 2264-2278

Jokar Arsanjani J, Vaz E, Bakillah M, Mooney P (2014) Towards initiating OpenLandMap founded on citizens' science: the current status of land use features of OpenStreetMap in Europe. In: Huerta, Schade, Granll (eds) Proceedings of the AGILE'2014 international conference on geographic information science, Castellón, AGILE Digital Editions: Castellón, Spain, 3-6 June 2014

Jokar Arsanjani J, Helbich M, Bakillah M, Loos L (2015a) The emergence and evolution of OpenStreetMap: a cellular automata approach. Int J Digit Earth 8(1):74-88. doi:10.1080/ 17538947.2013.847125

Jokar Arsanjani J, Bakillah M, Arsanjani JJ (2015b) Understanding the potential relationship between the socio-economic variables and contributions to OpenStreetMap. Int J Digit Earth 0:1-16. doi:10.1080/17538947.2014.951081

Jokar Arsanjani J, Vaz E (2015c) An assessment of a collaborative mapping approach for exploring land use patterns for several European metropolises. Int J Appl Earth Obs Geoinf 35:329-337. doi:10.1016/j.jag.2014.09.009

Klonner C, Barron C, Neis P, Höfle B (2014) Updating digital elevation models via change detection and fusion of human and remote sensor data in Urban environments. Int J Digit Earth $1-19$

Lauer J, Richter L, Ellersiek T, Zipf A (2014) TeleAgro+ - Analysis framework for agricultural telematics data, IWCTS '14, SIGSPATIAL'14, 4-7 Nov 2014, Dallas/Fort Worth, TX, USA

Mooney P, Corcoran P (2012) Characteristics of heavily edited objects in OpenStreetMap. Future Internet 4:285-305

Mooney P, Corcoran P (2013a) Analysis of interaction and co-editing patterns amongst OpenStreetMap contributors. Trans GIS

Mooney P, Corcoran P, Ciepluch B (2012) The potential for using volunteered geographic information in pervasive health computing applications. J Ambient Intell Humaniz Comput 1-15 (LA-English)

Mooney P, Rehrl K, Hochmair H (2013) Action and interaction in volunteered geographic information: a workshop review. J Locat Based Serv 7:291-311

Neis P (2014) Measuring the reliability of wheelchair user route planning based on volunteered geographic information. Trans GIS n/a-n/a, doi:10.1111/tgis.12087

Neis P, Zipf A (2012) Analyzing the contributor activity of a volunteered geographic information project - the case of OpenStreetMap. ISPRS Int J Geo-Inf 1:146-165 
Neis P, Singler P, Zipf A (2010) Collaborative mapping and emergency routing for disaster logistics-case studies from the haiti earthquake and the UN Portal for Afrika. In: Proceedings of Geospatial Crossroads @ GI_Forum '10, Salzburg, Austria, 6-9 July 2010

Over M, Schilling A, Neubauer S, Zipf A (2010) Generating web-based 3D city models from OpenStreetMap: the current situation in Germany. Comput Environ Urban Syst 34:496-507

Roick O, Hagenauer J, Zipf A (2011) OSMatrix - grid-based analysis and visualization of OpenStreetMap. In: State of the Map EU 2011, Vienna, Austria

Rylov M, Reimer A (2014) A comprehensive multi-criteria model for high cartographic quality point-feature label placement cartographica. Int J Geogr Inform Geovis, doi:10.3138/carto.49. 1.2137

Rylov M, Zipf A (2012) Solutions for limitations in label placement in OGC symbology encoding (SE) specification. In: Geoinformatik 2012, Heidelberg

Schilling A, Over M, Neubauer S, Neis P, Walenciak G, Zipf A (2009) Interoperable location based services for 3D cities on the Web using user generated content from OpenStreetMap. In: UDMS 2009. 27th Urban Data Management Symposium. Ljubljana, Slovenia

Schmitz S, Zipf A, Neis P (2008) New applications based on collaborative geodata - the case of routing. In: Proceedings of XXVIII INCA International Congress on Collaborative Mapping and Space Technology

Zielstra D, Zipf A (2010) Quantitative studies on the data quality of OpenStreetMap in Germany 